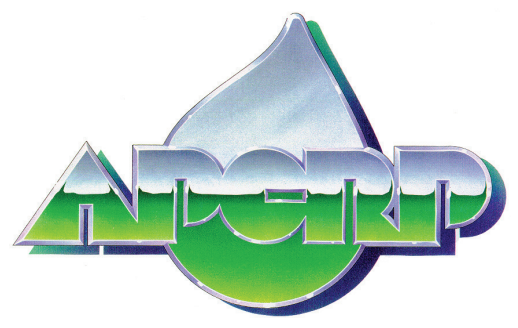

ERDC/TN APCRP-BC-43

September 2020

\title{
A First Examination of the Interaction between Alternaria alternantherae and Agasicles hygrophila on Alternanthera philoxeroides
}

by Nathan Harms and Judy F. Shearer

PURPOSE: The use of an alligatorweed (Alternanthera philoxeroides) insect biological control agent, alligatorweed flea beetle (Agasicles hygrophila Selman and Vogt), and the leaf spot plant pathogen, alligatorweed leaf pathogen (Alternaria alternantherae Holcomb and Antonopoulis), may provide enhanced control of alligatorweed infestations if competitive interactions between agents are minimal and damage to host plants are synergistic. However, to assess suitability of co-use of these two agents, it is necessary to first identify whether introduction of one may impact performance of the other. This technical note details a first examination of competitive interactions between A. hygrophila and A. alternantherae under varied temperature and host nutritional conditions. Presented are the results of laboratory experimentation and recommendations for future research.

INTRODUCTION: Biological control of A. philoxeroides in the United States was initiated in the 1960s, resulting in the introduction of three insect biological control agents (Buckingham 1996). The most successful and widely distributed agent is the alligatorweed flea beetle, Agasicles hygrophila (Figure 1). Although the alligatorweed flea beetle has suppressed alligatorweed growth and spread in most southern infestations, northern alligatorweed infestations in Arkansas, Tennessee, Alabama, and North Carolina remain unimpacted by biological control despite attempts to introduce cold-tolerant strains of A. hygrophila from more temperate areas of its native range in Argentina (Buckingham et al. 1983). Given the limitations of biological control use in the northern range of the plant, novel and innovative solutions are needed to reduce $A$. philoxeroides impacts in those areas.

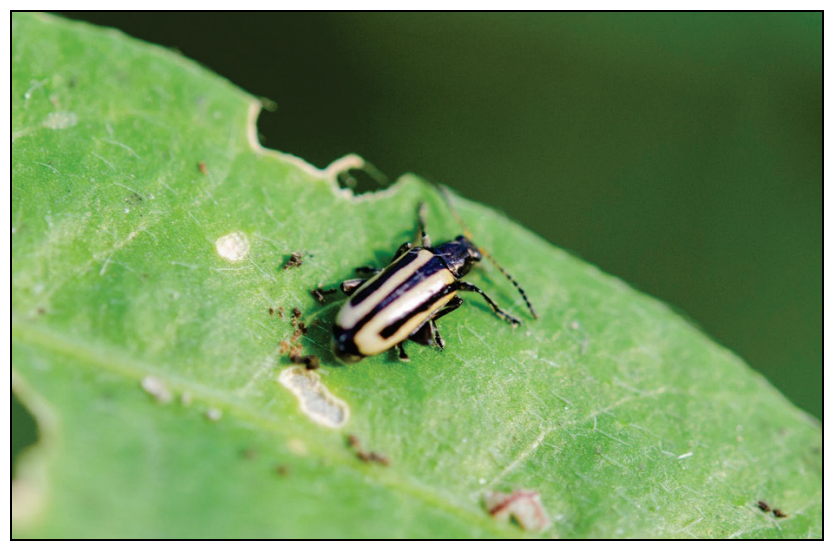

Figure 1. The alligatorweed flea beetle, Agasicles hygrophila, feeding on alligatorweed. 
In 1976, a fungal pathogen identified as Alternaria alternantherae (Figure 2) was discovered on A. philoxeroides plants near Baton Rouge, LA (Holcomb and Antonopoulos 1976). The fungus develops small purple lesions on leaves, which become necrotic over time, leading to leaf senescence and abscission from the plant (Holcomb 1978). Although A. alternantherae can be quite damaging when infection rate is high, it was found to infect a number of ornamental species, so its value as a classical biological control agent is equivocal (Holcomb 1978; Pomella et al. 2007). However, the combined use of pathogen application and introduction of the flea beetle has not been attempted, though they are commonly observed affecting the same plants in field settings (Harms and Shearer 2019).

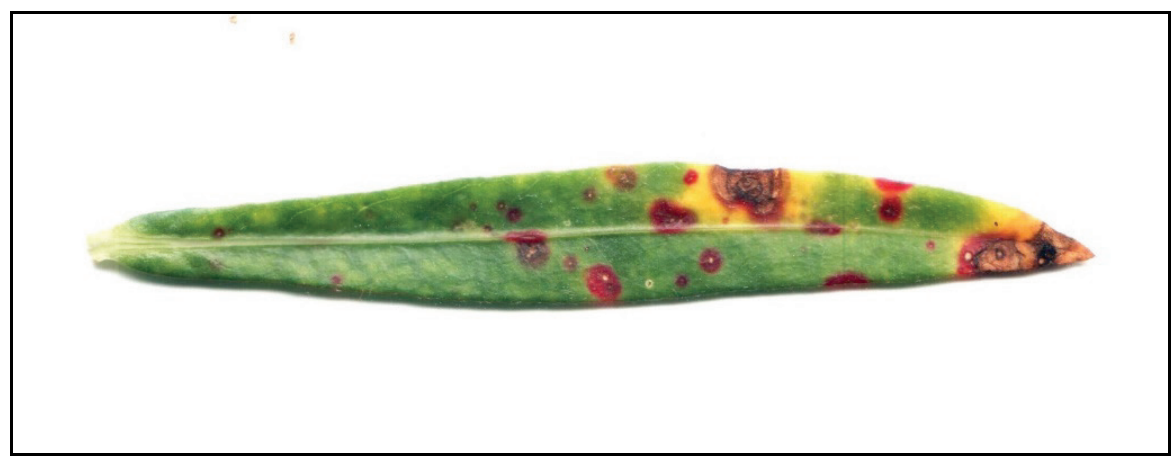

Figure 2. An A. philoxeroides leaf infected by the pathogenic fungus Alternaria alternantherae.

Because $A$. alternantherae appears to have a broad geographic distribution in the United States and is not limited to warmer climates (like $A$. hygrophila), it may be possible to use $A$. alternantherae in combination with annual introductions of the flea beetle to provide control of northern infestations. However, first it is necessary to determine (1) whether there is a competitive interaction between the two agents and (2) what is the strength of competition (i.e., how much is agent performance reduced by presence of the other). A strong competitive interaction (i.e., antagonism) might result in a reduction of $A$. philoxeroides suppression relative to that provided by either agent individually.

The literature on interactions between plant fungi and insect herbivores, particularly biological control agents, is sparse but largely consists of studies of direct or indirect interactions (Hatcher 1995). Direct interactions between insects and plant pathogenic fungi consist of a direct impact of one on the other, such as a change in insect performance due to feeding on fungi during consumption of infected leaf tissue (i.e., insect performance differences are fungal related and not directly related to leaf nutrition). In contrast, feeding by insects creates wounds that may make a plant more susceptible to fungal infection. Indirect interactions often involve some change in plant quality, which mediates the interaction between insect and fungus. For example, changes in nutritional quality of an infected plant, separate from the nutrition of the fungus itself, may impact feeding choices or development of herbivorous insects. Similarly, prolonged feeding by insects can affect plant quality, and resistance to disease, as the host plant allocates resources towards defense against herbivory or to compensatory regrowth. In the former scenario, fungal infection may increase because the enzymatic pathways used by plants to defend against herbivory and disease are mutually exclusive or represent tradeoffs (Thaler et al. 1999; Felton and Korth 2000) depending on the form of damage experienced by the plant. When insect feeding or other herbivory leads to compensatory regrowth, susceptibility to fungal infection may increase if nutritional quality of the regrowth is sufficient but levels of defensive chemicals are low. It is unclear which type of interaction (direct or 
indirect) is most common, and many studies of interactions may not actually determine whether observed outcomes are due to direct or indirect interactions but document the pattern, not the processes creating it. There are numerous possible outcomes from insect-fungal interactions mediated by a shared host plant, but the case of interacting biological control agents is particularly important because it may lead to improved control of economically and ecologically damaging target plants.

The objective of this work was to conduct initial experimentation to determine whether $A$. hygrophila and A. alternantherae are compatible and can co-exist by measuring their interaction on A. philoxeroides. Although the experiment was designed to measure the competitive outcome between agents, direct and indirect effects were not separated. This technical note presents the findings and gives recommendations for additional efforts to improve $A$. philoxeroides control in areas where it is needed.

\section{MATERIALS AND METHODS}

Plant Culture. Plants were collected from Baton Rouge, LA (N 30.3465', W 91.1394'), in 2015 and cultured in a greenhouse at the U.S. Army Engineer Research and Development Center (ERDC), Vicksburg, MS, under two levels of nitrogen. Plants were cultured under low (2 milligrams per liter $[\mathrm{mg} / \mathrm{L}] \mathrm{N})$ and high $(200 \mathrm{mg} / \mathrm{L} \mathrm{N})$ nitrogen-enriched nutrient media, which had previously produced $A$. philoxeroides with foliar nitrogen levels of $2.3 \pm 0.1$ milligrams per gram $(\mathrm{mg} / \mathrm{g}) \mathrm{N}$ (low) and $6.5 \pm 0.1$ $\mathrm{mg} / \mathrm{g}$ (high) (Mean \pm Standard Error [SE]; Harms and Cronin [2019]). The nutrient media was developed by modifying Hoagland's nutrient solution (Hoagland and Arnon 1950) to have zero nitrogen, then amending as needed with ammonium nitrate $\left(\mathrm{NH}_{4} \mathrm{NO}_{3}\right)$ to reach desired treatment levels. Plants were cultured for 8 weeks under these conditions, with nutrient media exchanged weekly. Four days prior to the experiment, approximately 250 milliliter $(\mathrm{mL})$ of fine grained sand was placed in 1-L plastic tubs, and then $250 \mathrm{~mL}$ of nutrient solution was added. Apical sections $(\sim 10$ centimeters [cm] in length) of greenhouse-cultured A. philoxeroides plants (the upper four leaf pairs) were cut and placed in the experimental containers and allowed to root before the experiment commenced. Nutrient solutions were replaced prior to the pathogen treatment being applied.

Pathogen Isolation. Alternaria alternantherae was isolated and cultured from A. philoxeroides plants collected at Blind River, LA (N 30.0949 ${ }^{\circ}$, W $90.7785^{\circ}$ ), during 2016. Diseased leaves were examined under a stereoscope, and a small section (approximately $0.5 \mathrm{~cm} \times 1.0 \mathrm{~cm}$ ) was excised from unique lesions, surfaced sterilized in 10\% sodium hypochlorite solution for 1 minute, rinsed in sterile water, and plated onto Martin's agar (Dhingra and Sinclair 1995) (maximum of three leaf pieces per plate). Plates were incubated in the dark at $25^{\circ} \mathrm{C}$ for 1 week. From the leading edge of distinct colonies, a small section $(1$ millimeter $[\mathrm{mm}] \times 1 \mathrm{~mm})$ was cut and placed onto a potato dextrose agar (PDA) (Difco, Detroit, MI) slant. The isolates were again allowed to grow for 1 week at $25^{\circ} \mathrm{C}$ at which time they were separated into morphotype, enumerated, sorted based on colony morphology and color. Each morphotype was identified to species when possible by plating it onto PDA and potato carrot agar (PCA) plates. Two different agars were used because PCA stimulates sporulation and PDA provides morphological characteristics (distinctive colony colors and growth patterns). Isolates were then accessed into the Biomanagement culture collection at ERDC.

Inoculum Preparation. Alternaria alternantherae (WES022516) was retrieved from storage and plated onto petri dishes containing PDA. The cultures were allowed to grow 2 weeks at room 
temperature $\left(21-22^{\circ} \mathrm{C}\right)$. One plate of the $A$. alternantherae culture was cut into small pieces $(1 \mathrm{~mm}$ $\times 1 \mathrm{~mm}$ ) and placed in two $250 \mathrm{~mL}$ flasks containing $100 \mathrm{~mL}$ of Richard's V-8 broth (10 grams [g]; $\mathrm{KNO}_{3}, 10 \mathrm{~g}$; $\mathrm{CaCO}_{3}, 3 \mathrm{~g}$; V-8 juice (Campbells, Camden, NJ), $200 \mathrm{~mL} ; \mathrm{H}_{2} \mathrm{O}, 800 \mathrm{~mL}$ ). The flasks were placed on a platform shaker (New Brunswick, Edison, NJ) set at 200 rotations per minute. Flasks were swirled daily to prevent fungal buildup along the sides of the flasks. After 7 days incubation, the contents of each flask were combined in a blender and ground 20 seconds to homogenize the culture. A $1 \mathrm{~mL}$ aliquot was added to a $9 \mathrm{~mL}$ sterile water blank and sequentially diluted to $1 \times 10^{6}$. Dilutions of $1 \times 10^{6}$ and $1 \times 10^{5}$ were plated onto Martin's agar (three plates per dilution), allowed to grow until distinct colonies were evident on the plate, and enumerated.

Insect Collection. Agasicles hygrophila adults were collected from the same location as $A$. alternantherae (Blind River) and cultured for a generation before the experiment. First-generation adults were allowed to oviposit on healthy plants; then egg masses were isolated in petri dishes and observed daily for hatching. Once sufficient numbers of neonates were available, the experiment commenced. Approximately 1 month passed from field collection of adults before eggs were collected.

Experimental Setup. The experimental design consisted of a factorial arrangement of the following treatment variables: nitrogen (low, high), competition (A. alternantherae alone, A. hygrophila alone, or both agents together), and temperature $\left(23^{\circ} \mathrm{C}\right.$ or $\left.30^{\circ} \mathrm{C}\right)$. Plants assigned to a treatment with $A$. alternantherae (A. alternantherae alone or A. alternantherae plus $A$. hygrophila) were inoculated with the pathogen first, then insects were added 24 hours later. This time lag allowed the fungal inoculum to dry on the plant and not have a physical impact on A. hygrophila larvae. Two upper leaves on each plant were pricked at the leaf axil with a fine insect pin ( 00 gauge), and then a single drop $(10 \mu \mathrm{l})$ of inoculum was applied at the rate of $1 \times 10^{6}$ colony forming units $\mathrm{mL}^{-1}$ to each wound. Plants were then placed in one of two reach-in environmental growth chambers at $23{ }^{\circ} \mathrm{C}$ or $30{ }^{\circ} \mathrm{C}$ until the next day. The following day, neonates $(<24$ hours old) were added to plants. Five neonates were gently placed on each plant with a soft-bristle paint brush; then fine-mesh (500 um) insect netting was secured over the tops of experimental containers with elastic bands. Within each environmental growth chamber, containers were placed on one of two shelves. To reduce variation and account for a shelf-effect (the lower shelf received reduced light), shelf was treated as a block in the statistical model. Experimental containers were randomly placed by shelf and chamber within each temperature treatment. There were six replicate containers per treatment combination.

The experiment continued until $A$. hygrophila adults were fully emerged, 21 days. Containers were then carefully examined, and the following response variables were determined: percent larval survival ([entrance holes in stem/number of larvae at the beginning of the experiment] *100), percent pupal survival ([number of entrance holes/by the number of exit holes] $* 100$ ), overall percent survival ([number of emerged adults/number of larvae at the beginning of the experiment] *100), and number of leaf lesions produced as a result of $A$. alternantherae infection.

Statistical Approach. To test whether nitrogen, temperature, and competition affect agent performance, mixed-effects models were used. Nitrogen (low, high), temperature $\left(23^{\circ} \mathrm{C}\right.$ or $30^{\circ} \mathrm{C}$ ), and competition (A. alternantherae, A. hygrophila, A. alternantherae + A. hygrophila) were treated as fixed effects, and shelf (block) was a random effect. Separate models were used for each response variable (A. hygrophila larval survival, A. hygrophila pupal survival, A. hygrophila total survival, number of leaf lesions). For each test, treatments were crossed in a fully factorial design, with the exception of the 
competition treatment. When testing for effects on insect performance, the A. alternantherae-alone treatment was excluded from analysis. Likewise, when testing for effects on pathogen performance, the A. hygrophila-alone treatment was excluded. To better meet assumptions of normality and to homogenize variances, survival data were arcsine square-root transformed, and leaf lesion data were log-transformed prior to analysis (Gotelli 1995), but raw data are presented here. Results are significant at $\alpha$ of 0.05 . Statistical tests were performed using Statistica version 12 (Statsoft).

RESULTS AND DISCUSSION: Both $A$. hygrophila and A. alternantherae were significantly affected by temperature and nitrogen but not by the interaction with the other agent (Table 1; Figures 3-4). Overall, A. hygrophila survival was significantly reduced with increased temperature and reduced nitrogen (Figure $3 \mathrm{c}$ ). Larval survival was affected primarily by nitrogen $(\mathrm{p}<0.01)$, with survival to pupation reduced by $70 \%$ in low-nitrogen treatments relative to high-nitrogen treatments (Figure 3a). In contrast, pupal survival, measured by the percentage of pupae surviving to emerge as adults, was primarily affected by temperature $(\mathrm{p}=0.02$; Figure $3 \mathrm{c})$. Pupal survival was $42 \%$ lower at $30{ }^{\circ} \mathrm{C}$ relative to $23^{\circ} \mathrm{C}$. This result is not surprising because both temperature and nitrogen influence on larval development is the subject of a separate study (Harms and Cronin 2019) in which it was determined survival decreases as temperature increases towards $30^{\circ} \mathrm{C}$, but those negative effects may be somewhat tempered by high foliar nitrogen levels. Additionally, nutrient acquisition occurs during larval development, so it is not surprising the largest nutrition-based effects would occur during that stage and not during pupal development. An interesting result was observed in the marginally statistically insignificant interaction between pupal survival and competition at low plant nitrogen $(\mathrm{p}=0.09)$ (Figure 3B). Pupal survival with low nitrogen was 1.6-fold higher with competition at low temperature and 12 -fold higher with competition at high temperature. The reason for a competitive effect at low but not high nitrogen is unclear. Plant nutrition-effects on insect biological control agents are well known (Forno and Bourne 1985; Room et al. 1989; Wheeler and Center 1996; Van Hezewijk et al. 2008; Center and Dray 2010) and have been implicated in both preventing establishment of agents when nutrition is poor and promoting establishment when nutrition is high (Room et al. 1989). Surprisingly though, alligatorweed has not received much attention in this regard, likely because early attempts at control were so successful. Control by $A$. hygrophila has been rapid and complete in most areas (Buckingham 1996). Some limited examination of alligatorweed nutrition occurred after it was recognized control could be variable, particularly in northern alligatorweed infestations, and it was believed site-specific conditions may limit effectiveness of the introduced agents if nutrient availability was low (Maddox and Rhyne 1975). The combined effects of foliar nitrogen and temperature on the development of $A$. hygrophila are of interest given the temporal variability of these two variables, which correspond with variable attack timing, especially in areas where the agent must immigrate annually because local overwintering conditions are harsh (Harms and Shearer 2017). 


\begin{tabular}{|c|c|c|c|c|c|c|c|c|c|c|c|c|}
\hline \multirow[b]{2}{*}{ Treatment } & \multicolumn{3}{|c|}{ Larval survival } & \multicolumn{3}{|c|}{ Pupal survival } & \multicolumn{3}{|c|}{ Overall survival } & \multicolumn{3}{|c|}{ Leaf lesions } \\
\hline & df & $\mathrm{F}$ & $p$ & df & $\mathrm{F}$ & $p$ & $\mathrm{df}$ & $\mathrm{F}$ & $p$ & df & $\mathrm{F}$ & $p$ \\
\hline $\mathrm{T}$ & 1 & 3.67 & 0.06 & 1 & 6.09 & 0.02 & 1 & 20.48 & $<0.01$ & 1 & 7.81 & 0.01 \\
\hline $\mathrm{N}$ & 1 & 25.72 & $<0.01$ & 1 & 0.07 & 0.79 & 1 & 28.54 & $<0.01$ & 1 & 11.95 & $<0.01$ \\
\hline C & 1 & 1.47 & 0.23 & 1 & 3.48 & 0.07 & 1 & 0.69 & 0.41 & 1 & 0.23 & 0.63 \\
\hline $\mathrm{T}^{*} \mathrm{~N}$ & 1 & 2.25 & 0.14 & 1 & 0.21 & 0.65 & 1 & 5.78 & 0.02 & 1 & 1.67 & 0.20 \\
\hline$T^{*} \mathrm{C}$ & 1 & 0.65 & 0.42 & 1 & 0.23 & 0.63 & 1 & 0.44 & 0.51 & 1 & 1.71 & 0.20 \\
\hline $\mathrm{N}^{*} \mathrm{C}$ & 1 & 0.04 & 0.84 & 1 & 3.06 & 0.09 & 1 & 2.77 & 0.10 & 1 & 0.29 & 0.59 \\
\hline $\mathrm{T}^{\star} \mathrm{N}^{*} \mathrm{C}$ & 1 & 0.00 & 0.97 & 1 & 0.58 & 0.45 & 1 & 1.03 & 0.31 & 1 & 0.01 & 0.92 \\
\hline Error & 66 & & & 34 & & & 68 & & & 67 & & \\
\hline
\end{tabular}

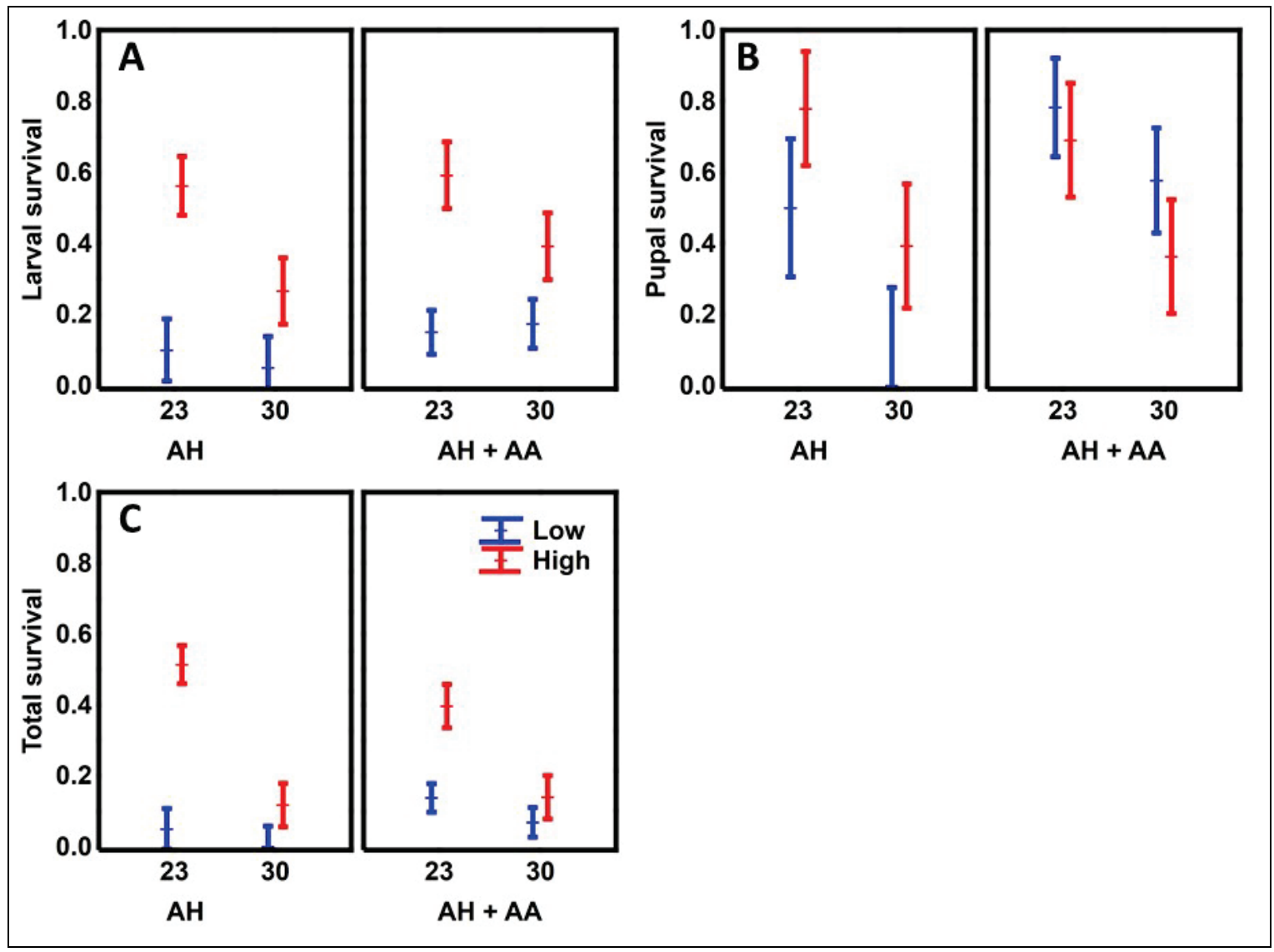

Figure 3. Mean ( $\pm \mathrm{SE})$ proportion of Agasicles hygrophila surviving (a) to the pupal stage, (b) pupae to adult emergence, (c) overall development from neonate to adult, in all treatment combinations. (AH=Agasicles hygrophila; AA=Alternaria alternantherae) 
The effect of plant quality on infection and performance of $A$. alternantherae had not been previously investigated. Although there was no competitive effect detected $(\mathrm{p}=0.63)$, significant effects of both nitrogen $(p<0.01)$ and temperature $(p=0.01)$ were detected. However, it is likely different mechanisms are behind the influence of each treatment. For example, the number of leaf lesions increased from high to low nitrogen levels. The first likely explanation for this pattern is alligatorweed plants under nutrient-limitation are more susceptible to infection. However, although there is evidence various types of physiological stress (e.g., from water-limitation, temperature) reduce the ability of the plant to resist infection, evidence to support enhanced infection rates in nitrogen-limited plants is equivocal and species specific (Snoeijers et al. 2000). In many cases, increased nitrogen availability leads to higher infection rates and increased disease symptoms but may depend on the form of available nitrogen (i.e., nitrate vs. ammonia) (Huber and Watson 1974). In contrast, early blight in many plant species caused by Alternaria solani is increased when nitrogen is limited (Thomas 1948; Agrios 2005). Given the fungus used in the current experiment is a close relative of $A$. solani, which is known to produce increased disease under low nutrient conditions, it is probable the pattern observed here is not anomalous. Under the temperature treatment of the experiment, high temperature produced fewer lesions than low temperature, which likely involves temperature limitations of the fungus rather than improved resistance to infection by the plant under elevated temperatures. Since temperature-related effects on A. alternantherae have been previously observed ${ }^{1}$ their presence during the current study was not surprising. The implications of these results are that infection of alligatorweed in the field may tend towards increased infection late in the year (autumn) when both temperatures and plant nutrition decrease (Harms, personal observation). Alternatively, if feeding by A. hygrophila reduces foliar nutrition through repeated defoliation/regrowth cycles, increased infection rates may be observed at other times of the year (i.e., spring).

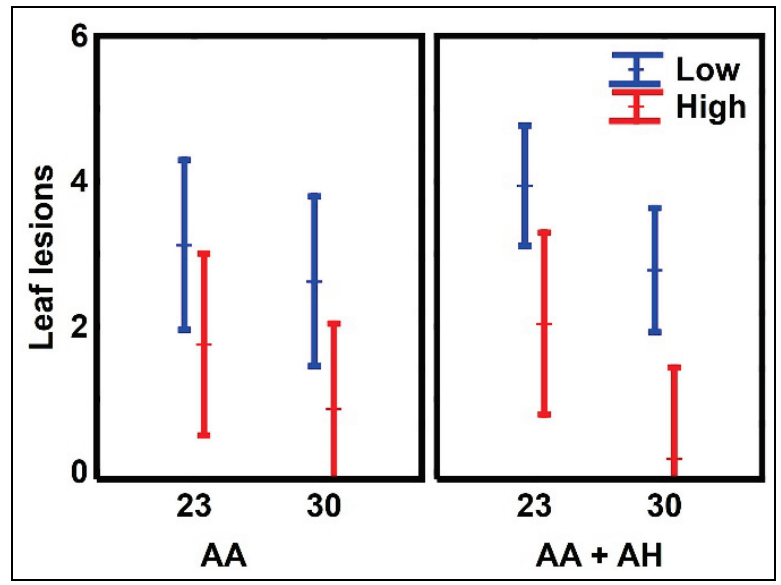

Figure 4. Mean $( \pm \mathrm{SE})$ number of leaf lesions in all treatment combinations. $(\mathrm{AA}=$ Alternaria alternantherae; $\mathrm{AH}=$ Agasicles hygrophila $)$

${ }^{1}$ J. F. Shearer. Personal observation. February 2017. ERDC Environmental Laboratory, Vicksburg, MS. 
This work represents an initial attempt to measure the interaction between an insect and fungal pathogen agent for the control of alligatorweed. Although a significant competitive effect was not detected, additional studies could further explore this possibility. First, because the replication was low $(n=6)$ due to limited space in controlled-environment growth chambers, access to additional chambers would increase the sample size and ability to detect significant competitive effects if they exist. Although a direct interaction appears to be minimal, the potential for indirect interactions between agents is strong. Agasicles hygrophila completely defoliates plants in southern areas, reducing or removing the primary substrate for infection by A. alternantherae. Repeated defoliation during the year likely reduces leaf nutrition as stored resources are depleted with each subsequent regrowth event. This seasonal change in plant nutrition, mediated by herbivory, could explain trends in A. alternantherae infection observed at field sites (Harms and Shearer 2019). Even without herbivory, A. philoxeroides protein and carbohydrate concentrations vary during the year, a phenomenon which has been discussed for its implications towards management timing (Weldon and Blackburn 1969; Pesacreta and Luu 1988).

Future research should examine longer-term effects of competition between $A$. hygrophila and $A$. alternantherae. This study was relatively short (3 weeks) and spanned a single generation of $A$. hygrophila. If plant infection influences insect performance, this may manifest as reduced body size or fecundity, with potential impacts on population development of $A$. hygrophila. Likewise, longterm feeding and defoliation by $A$. hygrophila may have competing negative effects on the ability of $A$. alternantherae to sustain and spread within and among plants and positive effects through reduced foliar nutrition and increased susceptibility to infection. Additionally, this study did not focus on the combined effects of insect feeding and fungal infection on growth and spread of $A$. philoxeroides plants, but the combined use of both agents is warranted since these results suggest a negative competitive effect is unlikely.

\section{ACKNOWLEDGEMENTS}

The authors express appreciation to David Lattuca for laboratory assistance and to Christopher Mudge and Aaron Schad for review of this technical note. This work was funded by the US Army Corps of Engineers, Aquatic Plant Control Research Program, under the leadership of Dr. Linda Nelson.

\section{REFERENCES}

Agrios, G. N. 2005. Plant Pathology. 5th edition. London: Academic Press.

Buckingham, G. R. 1996. "Biological Control of Alligatorweed, Alternanthera philoxeroides, the World's First Aquatic Weed Success Story.” Castanea 61(3): 232-243.

Buckingham, G. R., D. Doucias, and R. Theriot. 1983. "Reintroduction of the Alligatorweed Flea Beetle (Agasicles hygrophila Selman and Vogt) into the United States from Argentina." Journal of Aquatic Plant Management 21: 101-102.

Center, T. D., and F. A. Dray. 2010. "Bottom-Up Control of Water Hyacinth Weevil Populations: Do the Plants Regulate the Insects?” Journal of Applied Ecology 47: 329-337.

Dhingra, O. D., and J. B. Sinclair. 1995. Basic Plant Pathology Methods. Boca Raton, FL: CRC Press.

Felton, G. W., and K. L. Korth. 2000. “Trade-Offs between Pathogen and Herbivore Resistance." Current Opinion in Plant Biology 3: 309-314. 
Forno, I., and A. Bourne. 1985. "Feeding by Adult Cyrtobagous salviniae on Salvinia molesta under Different Regimes of Temperature and Nitrogen Content and the Effects on Plant Growth." BioControl 30: 279-286.

Gotelli, N. J. 1995. A Primer of Ecology. Sunderland, MA: Sinauer Associates Incorporated.

Harms, N. E., and J. T. Cronin. 2019. "Variability in Weed Biological Control: Effects of Foliar Nitrogen On Larval Development and Dispersal of the Alligatorweed Flea Beetle, Agasicles hygrophila." Biological Control 135: 16-22.

Harms, N. E., and J. F. Shearer. 2017. "Early-Season Dynamics of Alligatorweed Biological Control by Agasicles hygrophila in Louisiana and Mississippi." Journal of Aquatic Plant Management 55: 89-95.

Harms, N. E., and J. F. Shearer. 2019. "Spatial and Temporal Variability of the Alligatorweed Pathogen, Alternaria alternantherae, in Louisiana." Southeastern Naturalist 18(3): 441-450.

Hatcher, P. E. 1995. "Three-Way Interactions between Plant Pathogenic Fungi, Herbivorous Insects, and Their Host Plants." Biological Reviews 70: 639-694.

Hoagland, D. R., and D. I. Arnon. 1950. The Water-Culture Method for Growing Plants without Soil. Circular. California Agricultural Experiment Station 347.

Holcomb, G. 1978. "Alternaria alternantherae from Alligatorweed Also is Pathogenic on Ornamental Amaranthaceae species." Phytopathology 68: 265-266.

Holcomb, G. E., and A. A. Antonopoulos. 1976. “Alternaria alternantherae: A New Species Found on Alligatorweed. Mycologia 68: 1125-1129.

Huber, D., and R. Watson. 1974. "Nitrogen Form and Plant Disease." Annual Review of Phytopathology 12: 139-165.

Maddox, D., and M. Rhyne. 1975. "Effects of Induced Host-Plant Mineral Deficiencies on Attraction, Feeding, and Fecundity of the Alligatorweed Flea Beetle.” Environmental Entomology 4: 682-686.

Pesacreta, G. J., and K. T. Luu. 1988. Feasibility of Relating Phenology and Carbohydrate Partitioning to Improve Aquatic Plant Control. Miscellaneous Paper A-88-7. Vicksburg, MS: Department of the Army, Waterways Experiment Station, Corps of Engineers, Environmental Laboratory.

Pomella, A. W. V., R. W. Barreto, and R. Charudattan. 2007. Nimbya alternantherae a potential biocontrol agent for alligatorweed, Alternanthera philoxeroides. BioControl 52:271-288.

Room, P., M. Julien, and I. Forno. 1989. "Vigorous Plants Suffer Most from Herbivores: Latitude, Nitrogen and Biological Control of the Weed Salvinia molesta." Oikos 54: 92-100.

Snoeijers, S. S., A. Pérez-García, M. H. A. J. Joosten, and P. J. G. M. De Wit. 2000. "The Effect of Nitrogen on Disease Development and Gene Expression in Bacterial and Fungal Plant Pathogens." European Journal of Plant Pathology 106: 493-506.

Thaler, J. S., A. L. Fidantsef, S. S. Duffey, and R. M. Bostock. 1999. "Trade-Offs in Plant Defense against Pathogens and Herbivores: A Field Demonstration of Chemical Elicitors of Induced Resistance." Journal of Chemical Ecology 25: $1597-1609$.

Thomas, H. R. 1948. "Effect of Nitrogen, Phosphorus and Potassium on Susceptibility of Tomatoes to Alternaria solani." J. Agric. Res 76: 289-306.

Van Hezewijk, B. H., R. A. De Clerck-Floate, and J. R. Moyer. 2008. "Effect of Nitrogen on the Preference and Performance of a Biological Control Agent for an Invasive Plant." Biological Control 46: 332-340.

Weldon, L., and R. Blackburn. 1969. "Herbicidal Treatment Effect on Carbohydrate Levels of Alligatorweed.” Weed Science 17(1): 66-69.

Wheeler, G., and T. Center. 1996. "The Influence of Hydrilla Leaf Quality on Larval Growth and Development of the Biological Control Agent Hydrellia pakistanae (Diptera: Ephydridae).” Biological Control 7: 1-9.

NOTE: The contents of this technical note are not to be used for advertising, publication, or promotional purposes. Citation of trade names does not constitute an official endorsement or approval of the use of such products. 